\title{
ARTICLE
}

Received 5 May 2015 | Accepted 2 Nov 2015 | Published 17 Dec 2015

DOI: 10.1038/ncomms10107

OPEN

\section{Interplay between plasmon and single-particle excitations in a metal nanocluster}

Jie Ma1 ${ }^{1}$ Zhi Wang ${ }^{2} \&$ Lin-Wang Wang ${ }^{1}$

Plasmon-generated hot carriers are used in photovoltaic or photochemical applications. However, the interplays between the plasmon and single-particle excitations in nanosystems have not been theoretically addressed using $a b$ initio methods. Here we show such interplays in $\mathrm{Ag}_{55}$ nanocluster using real-time time-dependent density functional theory simulations. We find that the disappearance of the zero-frequency peak in the Fourier transform of the band-to-band transition coefficient is a hallmark of the plasmon. We show the importance of the $d$-states for hot-carrier generations. If the single-particle $d$-to-s excitations are resonant to the plasmon frequency, the majority of the plasmon energy will be converted into hot carriers, and the overall hot-carrier generation is enhanced by the plasmon; if such resonance does not exist, we observe an intriguing Rabi oscillation between the plasmon and hot carriers. Phonons play a minor role in plasmonic dynamics in such small systems. This study provides guidance on improving plasmonic applications.

\footnotetext{
${ }^{1}$ Joint Center for Artificial Photosynthesis and Materials Sciences Division, Lawrence Berkeley National Laboratory, Berkeley, California 94720, USA.

${ }^{2}$ Materials Sciences Division, Lawrence Berkeley National Laboratory, Berkeley, California 94720, USA. Correspondence and requests for materials should be addressed to L.-W.W. (email: Iwwang@lbl.gov).
} 
$\mathrm{P}$ lasmons are collective oscillations of the electron density, which can convert light energies into electronic excitations ${ }^{1,2}$. Due to the enhanced electromagnetic fields near metallic surfaces, metal nanostructures have been used in single-molecule spectroscopy, surface-enhanced Raman spectroscopy and surface-enhanced fluorescence ${ }^{3-5}$. For noble-metal nanostructures, the plasmon frequencies, which depend on the size, shape, metal composition and surrounding dielectric environment ${ }^{6,7}$, are generally in visible or ultraviolet regions that cover a large portion of the solar spectrum ${ }^{8}$. Due to the large oscillator strengths of plasmons, metal nanostructures can be used as antennas to absorb sunlight ${ }^{1,8,9}$. An intensely studied current topic is to use plasmons for photovoltaic or photochemical reactions. To realize those goals, the collective plasmon must be converted into single-particle excitations, that is, hot carriers, so that the carriers can be collected in solar cells or injected into chemical reactions ${ }^{9-16}$. However, some basic questions are yet to be answered. For example, how much light energy is absorbed by plasmons and hot carriers? Will all the plasmon energy convert to hot carriers and enhance the hotcarrier generation rates?

Plasmon modes can be simulated classically using the Drude model and Maxwell equations. Such calculations can yield accurate plasmon modes for systems down to tens of nanometres. However, when the system sizes shrink to the order of nanometres, the classical method could fail ${ }^{17,18}$ due to the lack of nonlocal response in the dielectric function and interband transitions. In such small systems, quantum mechanical descriptions, such as the linear-response theory, become necessary ${ }^{17,19-22}$. Quantum mechanically, the plasmon modes can be defined by the poles in the complex frequency-space $\omega$ of the inverse dielectric function $\varepsilon^{-1}\left(\mathbf{r}_{1}, \mathbf{r}_{2}, \omega\right)^{23}$; specifically, a plasmon mode $v\left(\mathbf{r}, \omega_{\mathrm{p}}\right)$ satisfies $\int \varepsilon\left(\mathbf{r}_{1}, \mathbf{r}_{2}, \omega_{\mathrm{p}}\right) v\left(\mathbf{r}_{2}, \omega_{\mathrm{p}}\right) \mathrm{d} \mathbf{r}_{2}=0$. Here the plasmon frequency $\omega_{\mathrm{p}}$ is a complex number, with its imaginary part indicating the plasmon life time. Unfortunately $\varepsilon^{-1}\left(\mathbf{r}_{1}, \mathbf{r}_{2}, \omega\right)$ does not provide a real-time picture of the interplays between the plasmon and hot carriers. There are several recent theoretical studies on the plasmon-induced hotcarriers generations, showing that hot carriers can be effectively excited by plasmons ${ }^{24,25}$. However, those studies treated plasmons classically as oscillating electromagnetic fields, and used the perturbation theory (Fermi's golden rule) to calculate the hot-carrier generations. They did not describe the feedback of hot carriers to plasmons, nor represent the plasmon and singleparticle excitations in a unified quantum mechanical framework.

The real-time ( $\mathrm{rt}$ ) time-dependent density functional theory (TDDFT) is a powerful tool to study the plasmonic processes.
The rt-TDDFT describes directly the Coulomb interactions between electrons, and its ability to describe plasmons has been demonstrated in many previous publications ${ }^{26-30}$. The rt-TDDFT was used to study jellium models or small systems such as one-dimensional atom chains ${ }^{26,27,30}$. There were also rt-TDDFT simulations on nanoclusters up to several hundred atoms using local-orbital basis or real-space grids ${ }^{28,29}$. However, those works studied only the optical absorption spectra ${ }^{28,29}$, instead of the interplays and energy transfers between the plasmon and single-particle excitations. For many interesting physical problems, including the plasmon decay, hot-carrier generations and carrier transports, long-time simulations (10-1,000 fs) are needed. Our recently developed fast rt-TDDFT algorithm ${ }^{31}$ enables us to simulate such long time with plane-wave basis sets.

In this work, we will use rt-TDDFT to study the plasmon dynamics in $\mathrm{a}_{55}$ nanocluster. The $\mathrm{Ag}_{55}$ nanocluster has been experimentally synthesized and its atomic structure is well determined ${ }^{32,33}$. A recent experiment measured the plasmon frequency of $3.8 \mathrm{eV}$ (ref. 34). Such small nanoclusters may have great potentials for photocatalysis due to high carrier-injection rates. We propose an unambiguous approach to distinguish the plasmon from single-particle excitations based on the band-toband transition coefficient. We find that if the single-particle $d$-to-s excitations resonant to the plasmon frequency exist, most plasmon energy will be converted into hot carriers, enhancing hot-carrier generations. However, if the resonances do not exist, the plasmon decays slowly and may exhibit Rabi oscillations. We propose that by modifying the electronic structures of nanoclusters (for example, the number of electrons, plasmon frequency, $d$-band energy and so on), the plasmon decay and hotcarrier generations can be tuned. The electron-phonon interactions play a minor role during the plasmon decay in $\mathrm{Ag}_{55}$.

\section{Results}

Laser excitations in $\mathbf{A g}_{\mathbf{5 5}}$. The calculated atomic structure and eigen energies of $\mathrm{Ag}_{55}$ are shown in Fig. 1a. The neutral $\mathrm{Ag}_{55}$ has 605 electrons and is an open-shell system. The 301st-303rd states are almost degenerated and partially occupied, and the 304th state is the lowest unoccupied state (LUMO). To study the excitations, we apply an external electric field $\mathbf{E}(t)=$ $\mathbf{E}_{0} \sin \left(\omega_{1} t\right) \exp \left(-t^{2} / 2 \sigma^{2}\right) / \sqrt{2 \pi} \sigma$ to mimic a laser pulse at the frequency $\omega_{1}$ with a Gaussian envelope. The optical absorption spectrum can be obtained from the time-dependent dipole response (the imaginary part of $\alpha(\omega)$ described in Methods section), and is shown in Fig. 1b. We observe a strong absorption peak at $\omega_{\mathrm{p}} \approx 3.6 \mathrm{eV}$, which is in good agreement with the recent
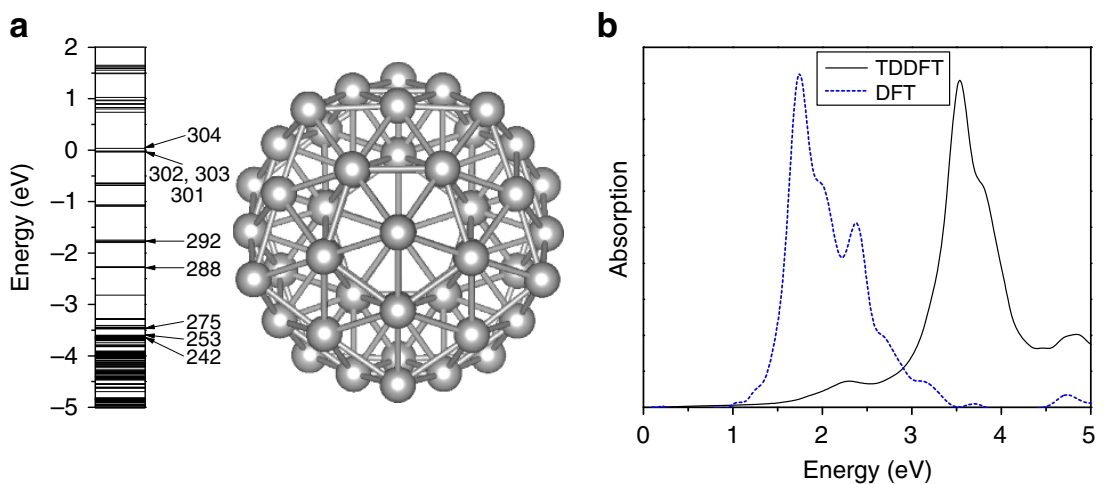

Figure 1 | The calculated eigen energies, atomic structure and absorption spectra of $\mathbf{A g}_{\mathbf{5 5}}$. (a) The calculated atomic structure and eigen energies of the icosahedral $\mathrm{Ag}_{55}$. The Fermi energy is set to zero. Several important states are labelled. Each silver ball represents a $\mathrm{Ag}$ atom. (b) The absorption spectra calculated by the rt-TDDFT (the black-solid line) and by the DFT eigen states (the blue-dashed line). The heights of the main peaks are normalized. 
experimental result of $3.8 \mathrm{eV}$ (ref. 34). We have also calculated the absorption spectrum from the DFT single-particle eigen states: $\sum_{i, j}\left|\left\langle\phi_{i}|\mathbf{r}| \phi_{j}\right\rangle\right|^{2} \delta\left(\omega_{j}-\omega_{i}-\omega\right)\left(1-O_{j}^{0}\right) O_{i}^{0}$, where $\phi_{i}, \omega_{i}$ and $O_{i}^{0}$ are the wave function, eigen energy and Fermi-Dirac occupation of the $i$ th eigen state, respectively. The resulting curve shows a main peak at $\sim 1.7 \mathrm{eV}$ (Fig. 1b). In all the calculations, we have modified the pseudopotential, so that the calculated Ag $d$-band energy agrees with the experiment ${ }^{35}$, which is shown in Supplementary Note 1 and Fig. 1.

To exam the resonant excitation, we set the laser frequency $\omega_{1}=\omega_{\mathrm{p}}$ with $\sigma=6 \mathrm{fs}$ and $\left|\mathbf{E}_{0}\right|=10^{-3}$ Hartree per Bohr. The electric field is along the $x$ axis, starting at $t=0 \mathrm{fs}$ and fading off after $\sim 10 \mathrm{fs}$. In response to this external electric field, the dipole moment of the $\mathrm{Ag}_{55}$ nanocluster oscillates along the $x$ axis, as shown in Fig. 2a. From 0 to $10 \mathrm{fs}$, as the electric field exists (the laser is on), the charge density is driven by the external electric field, and both the plasmon and single-particle modes have been excited. After $10 \mathrm{fs}$, as the external electric field fades off, the dipole oscillation continues by itself due to internal excitations. From 10 to $20 \mathrm{fs}$, the dipole oscillation amplitude is rather large. Two of the charge sloshing pictures $\Delta \rho(\mathbf{r}, t)=\rho(\mathbf{r}$, $t)-\rho_{0}(\mathbf{r})$ (where $\rho_{0}(\mathbf{r})$ is the ground-state charge density) at $t=19.2$ and $19.8 \mathrm{fs}$ are shown in Fig. $2 \mathrm{~b}$. The $\Delta \rho$ is localized on the surface, and sloshes from one side of the nanocluster to the other, fitting well with the classical picture of the surface plasmon. From 20 to $27 \mathrm{fs}$, the dipole oscillation amplitude decays to relatively small values. Two of the charge density differences $\Delta \rho$ at $t=38.0$ and $38.6 \mathrm{fs}$ are shown in Fig. 2b. The $\Delta \rho$ is not localized on the surface and does not slosh, which may suggest the plasmon decay qualitatively. Such dipole oscillations can be observed experimentally as demonstrated in a recent experiment by Mittal et al. ${ }^{36}$ Using optical pump and probe method, they observed the polarization oscillations up to $80 \mathrm{fs}$ in $\mathrm{Ag}$ nanoclusters. Their experiments confirmed the possibility of long-living dipole oscillations, similar to our results.

Distinguishing the plasmon mode. How to distinguish the collective plasmon excitation from the non-collective singleparticle excitation quantum mechanically is a challenging issue. In particular, for small molecules and nanostructures, the plasmon frequency $\omega_{\mathrm{p}}$ overlaps with the single-particle excitation energies, which makes their interplays interesting but difficult to analyse. There are several previous publications on this topic using different approaches ${ }^{11,23,37-43}$. The plasmon and single-particle excitations cannot be clearly distinguished from the absorption spectrum, because they both can have strong absorption peaks for small nanoclusters. Beck identified the plasmon using charge density oscillations ${ }^{43}$. However, the resonant but non-collective single-particle excitation from the $i$ th eigen state to the $j$ th eigen state also produces a charge density oscillation. This can be seen from the expansion of the time-dependent wave function $\psi_{i}(t)$ on the eigen states in a perturbation theory:

$$
\psi_{i}(t) \approx \exp \left(-i \omega_{i} t\right)\left[\phi_{i}+C_{j, i}(t) \exp \left(-i \Delta \omega_{j, i} t\right) \phi_{j}\right]
$$

and the charge density change is

$$
\Delta \rho(t) \approx C_{j, i}(t) \exp \left(-i \Delta \omega_{j, i} t\right) \phi_{i}^{*} \phi_{j}+c c .
$$

For resonant excitations, $\Delta \omega_{j, i}=\omega_{j}-\omega_{i}=\omega_{\mathrm{p}}$, so $\Delta \rho(t)$ also oscillates at the frequency $\omega_{\mathrm{p}}$. Thus, we cannot use the charge density (or dipole moment) oscillation alone to tell the difference. One can attempt to use the charge density plot, such as Fig. 2b, to say there may be stronger plasmon excitations at $19 \mathrm{fs}$ than those at $38 \mathrm{fs}$, but it is difficult to quantify the amounts of the plasmon and single-particle excitations. Krauter et al. $^{41}$ proposed to identify plasmons based on orbital transitions and momentum conservations, but their approach requires certain (quasi-)symmetry of the system. Other groups proposed to identify plasmons by scaling the electron-electron interaction $^{23}$. Although useful, this approach requires a serial of calculations on artificial systems. The occupation numbers on eigen states (that is, $\sum_{i}\left|C_{j, i}(t)\right|^{2} O_{i}^{0}$ ) were also used to identify plasmons ${ }^{30,40}$. However, the occupation change may be a mixture of both the plasmon and single-particle excitations, as we will show below. We find the transition coefficient $C_{j, i}(t)$, especially the Fourier transform of $C_{j, i}(t)$ (instead of $\left|C_{j, i}(t)\right|^{2}$, which loses the phase information), can be used to distinguish the plasmon from single-particle excitations in an unambiguous way.

Equations (1) and (2) are general formulas that apply for all transitions. However, the collective behavior of the plasmon must have caused unique feature of $C_{j, i}(t)$, compared with $C_{j, i}(t)$ of single-particle excitations. In the following we will take $j=304$
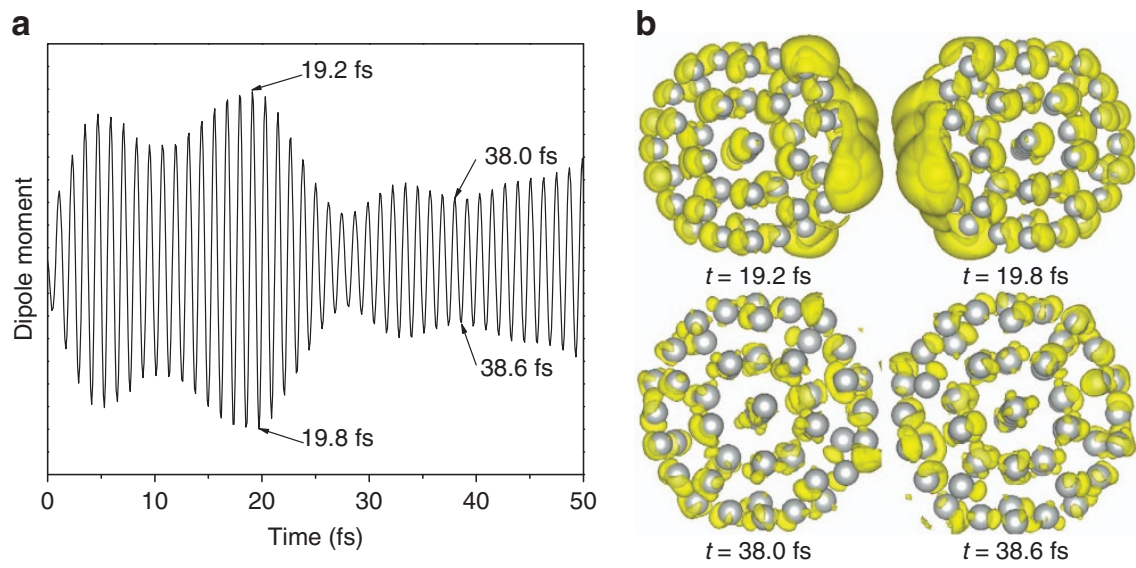

Figure 2 | The dipole moment and charge density oscillations. (a) The dipole moment oscillations as a function of time. (b) The charge density difference $\Delta \rho$ when the dipole moment achieves a local maximum or local minimum as labelled in $\mathbf{a}$. At $t=19.2$ and $19.8 \mathrm{fs}$, the dipole moment oscillation amplitude is large. The $\Delta \rho$ is localized on the surface, and sloshes from one side of the nanocluster to the other side, fitting well with the classical picture of the surface plasmon mode. When the dipole moment oscillation is decayed ( $t=38.0$ and $38.6 \mathrm{fs}$ ), the charge sloshing disappears, and $\Delta \rho$ is no longer localized on the surface. 
(LUMO) state as an example to discuss the behaviours of $C_{j, i}(t)$. Figure 3a shows $\left|C_{\mathrm{LUMO}, i}(t)\right|^{2}$ for all the transitions from the occupied states $(i \leq 303)$ to the LUMO state (the 304 th state). We observe two clearly distinct types of transitions. One type of $\left|C_{\mathrm{LUMO}, i}(t)\right|^{2}$ shows regular high-frequency synchronized oscillations (for example, $i=292$ ), and the other shows smooth amplitude variations (for example, $i=253$ ).

All the slowly varying transitions have nearly resonant energies $\omega_{\mathrm{LUMO}}-\omega_{i} \approx \omega_{\mathrm{p}}$ (for example, $\omega_{\mathrm{LUMO}}-\omega_{275}=3.5$, $\omega_{\text {LUMO }}-\omega_{253}=3.65$ and $\left.\omega_{\text {LUMO }}-\omega_{242}=3.7 \mathrm{eV}\right)$. The Fourier transform of $C_{\mathrm{LUMO}, 253}(t)$ (Fig. $3 \mathrm{C}$ ) shows only one peak around $\omega=0$. Thus, we have $C_{j, i}(t)=f(t)$, where $f(t)$ is a slowly varying (compared with $\omega_{\mathrm{p}}$ ) function, and equation (1) can be rewritten as:

$$
\psi_{i}(t) \approx \exp \left(-i \omega_{i} t\right)\left[\phi_{i}+f(t) \exp \left(-i \Delta \omega_{j, i} t\right) \phi_{j}\right]
$$

This is a typical independent single-particle excitation described by the single-particle time-dependent perturbation theory within the rotating-frame approximation. Only single-particle transitions with nearly resonant frequencies $\Delta \omega_{j, i} \approx \omega_{\mathrm{p}}$ can be effectively excited. The charge density oscillates at $\Delta \omega_{j, i} \approx \omega_{\mathrm{p}}$ due to the phase factor $\exp \left(-i \Delta \omega_{j, i} t\right)$. The single-particle excitations correspond to the so-called 'hot-carriers'.
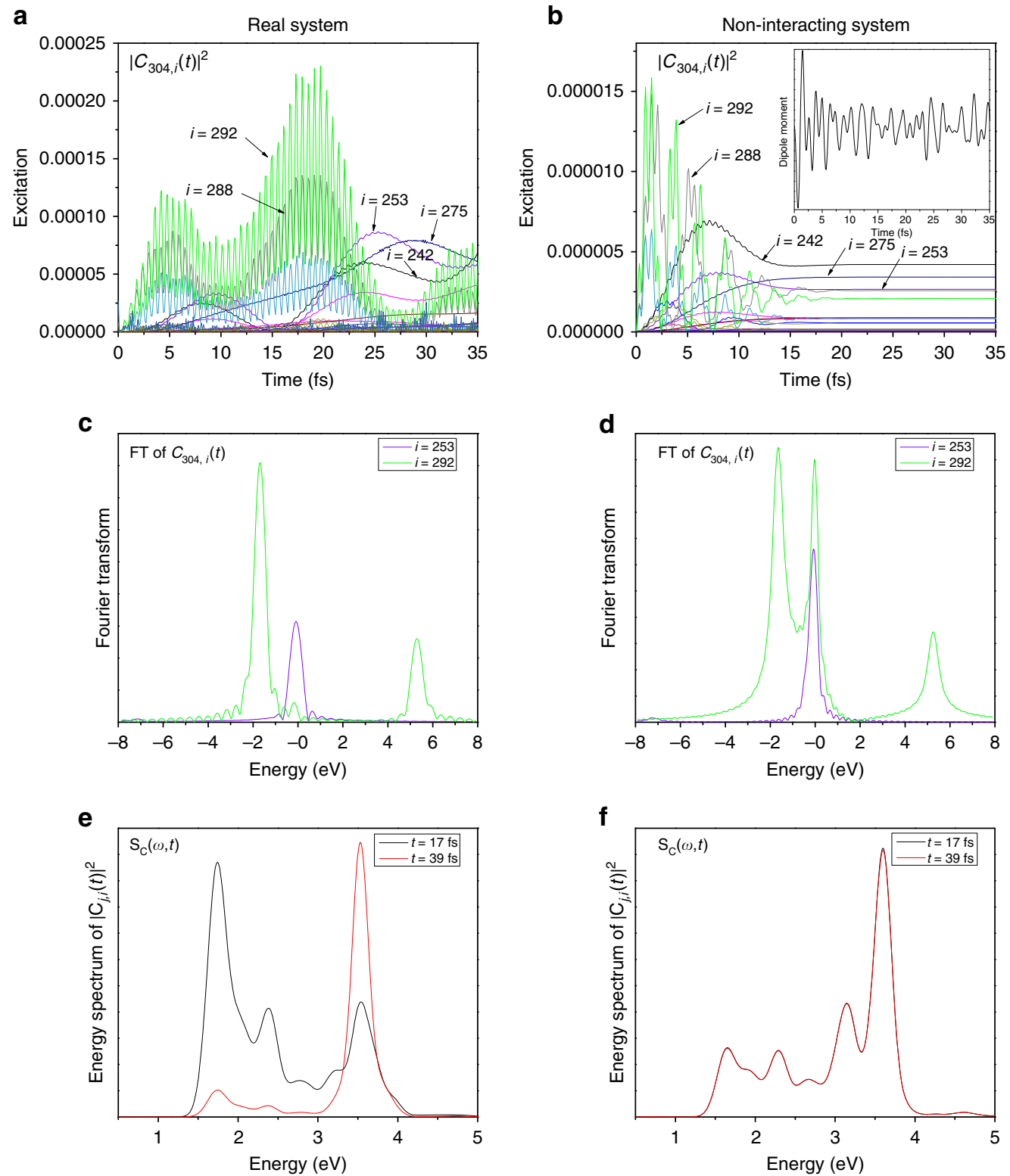

Figure 3 | The analysis of the transition coefficients $\boldsymbol{C}_{\boldsymbol{j}, \mathbf{i}}(\boldsymbol{t})$. We show $j=304$ (LUMO) state here. (a) The $\left|\boldsymbol{C}_{304, i}(t)\right|^{2}$ indicates the transition from the ith state to the LUMO (304th) state. Several important transitions are labelled. Two distinct types (rapidly oscillating and slowly varying) of transitions can be observed. (b) The $\left|C_{304, i}(t)\right|^{2}$ for the non-interacting system. The slowly varying transitions behave similarly to those in a but the rapidly oscillating ones are different. The inset shows that the dipole moment oscillates irregularly. (c) The Fourier transform (FT) of $C_{j, i}(t)$ for the real system. There is no peak at 0 for the rapidly oscillating transition $(i=292)$. (d) The FT of $C_{j, i}(t)$ for the non-interacting system. There is a peak at 0 for the oscillating transition $(i=292$ ). (e) The energy spectrum of $\left|C_{j, i}(t)\right|^{2}\left(S_{C}(\omega, t)\right)$ at $t=17$ and $39 \mathrm{fs}$ for the real system. At $t=17 \mathrm{fs}$, there are strong off-resonant peaks at 1.7 and $2.3 \mathrm{eV}$; at $t=39 \mathrm{fs}$ those peaks disappear and the resonant peak at $3.6 \mathrm{eV}$ is enhanced. (f) The $S_{C}(\omega, t)$ for the non-interacting system. The main peak is always at $3.6 \mathrm{eV}$ with no significant off-resonant peaks. 
In contrast, all the rapidly oscillating transitions have off-resonant energies $\omega_{\text {LUMO }}-\omega_{i}<\omega_{\mathrm{p}}$ (for example, $\omega_{\text {LUMO }}-\omega_{292}=1.78$ and $\left.\omega_{\text {LUMO }}-\omega_{288}=2.3 \mathrm{eV}\right)$, and the Fourier transform of $C_{\mathrm{LUMO}, 292}(t)$ shows two peaks at $\Delta \omega_{j, i} \pm \omega_{\mathrm{p}}$. Both the real and imaginary parts of $C_{\mathrm{LUMO}, 292}(t)$ (Supplementary Fig. 2 and Note 2) oscillate around zero, which confirms the disappearance of the zero-frequency peak. Because the Fourier transform has two peaks at $\Delta \omega_{j, i} \pm \omega_{\mathrm{p}}, C_{j, i}(t)=A$ $\exp \left[i\left(\Delta \omega_{j, i}+\omega_{\mathrm{p}}\right) t\right]+B \exp \left[i\left(\Delta \omega_{j, i}-\omega_{\mathrm{p}}\right) t\right]$, and it can be written as $e^{i \Delta \omega_{j, i} t} f(t) \sin \left(\omega_{\mathrm{p}} t+\theta\right)$. Here $A, B$ and $f(t)$ are slowly varying (compared with $\omega_{\mathrm{p}}$ ) functions, and $\theta$ is a complex phase factor. Thus, equation (1) can be rewritten as:

$$
\psi_{i}(t) \approx \exp \left(-i \omega_{i} t\right)\left[\phi_{i}+f(t) \sin \left(\omega_{\mathrm{p}} t+\theta\right) \phi_{j}\right]
$$

It cannot be described by the single-particle perturbation theory. It also does not require that the resonant condition $\Delta \omega_{j, i} \approx \omega_{\mathrm{p}}$, as $\Delta \omega_{j, i}$ does not appear in equation (4). The charge density oscillation (square of equation (4)) is contributed coherently from all the cross terms: $f(t) \sin \left(\omega_{\mathrm{p}} t+\theta\right) \phi_{i}^{*} \phi_{j}+c c$, with the same frequency $\omega_{\mathrm{p}}$. These synchronized off-resonant transitions constitute the plasmon mode. Since there is no resonant requirement for the plasmon-related transitions, many transitions with $\left|\omega_{i}-\omega_{j}\right|<\omega_{\mathrm{p}}$ can collectively and coherently construct the plasmon through electron-electron interactions. This contributes to the large plasmon oscillator strength, making it a strong light absorber. However, since the total oscillator strength is conserved, the non-collective single-particle $i$ to $j$ transitions are reduced. In Fig. 1b, compared with the strong single-particle excitations around $1.7 \mathrm{eV}$ in the DFT spectrum, the TDDFT spectrum around $1.7 \mathrm{eV}$ becomes much weaker. Most of their oscillator strengths have been shifted to the plasmon peak around $3.6 \mathrm{eV}$. The concept of plasmon as a collection of coherent transitions has long existed. Our analysis fits the picture well and provides direct evidence.

To further prove that the rapidly oscillating $C_{j, i}(t)$ comes from the plasmon, we have performed a time-dependent evolution on a non-interacting system with the same external electric field. In this simulation, we fix the potential in the Hamiltonian $V[\rho(\mathbf{r}, t), t]=V[\rho(\mathbf{r}, 0), t]$ (except the external potential) making it independent of the charge density. We would like to stress that this non-interacting system is an unrealistic system with no experimental counterpart. In this model, all the electrons are treated as independent single particles. Because the electron-electron interaction is zero, the plasmon excitation is impossible. Thus, the plasmon-related transitions should behave differently, while the single-particle transitions should remain the same.

The dipole moment of the non-interacting system (inset of Fig. 3b) shows an irregular behavior, compared with Fig. 2a. For the slowly varying transitions (for example, $i=253$ ), both the $\left|C_{\mathrm{LUMO}, i}(t)\right|^{2}$ (Fig. 3b) and the Fourier transform (Fig. 3d) are similar to those in Fig. 3a,c. However, for the rapidly oscillating transitions, the regular oscillations of $\left|C_{\mathrm{LUMO}, i}(t)\right|^{2}$ (for example, $i=292$ ) are replaced by irregular oscillations. The irregularly oscillating transitions decay with time, and almost disappear after the laser fades off. Besides the two peaks at $\Delta \omega_{i, j} \pm \omega_{\mathrm{p}}$ in Fig. $3 \mathrm{c}$, the Fourier transform of $C_{\mathrm{LUMO}, 292}(t)$ has a strong peak at $\omega=0$ in Fig. 3d. This can be explained by the independent singleparticle time-dependent perturbation theory ${ }^{44}$ under a $\sin \left(\omega_{\mathrm{p}} t\right)$ perturbation along the $x$ axis:

$$
C_{j, i}(t)=-\frac{\left\langle\phi_{j} \cdot|x| \phi_{i}\right\rangle}{2 i \hbar}\left\{\frac{\exp \left[i\left(\Delta \omega_{j, i}+\omega_{\mathrm{p}}\right) t\right]-1}{\Delta \omega_{j, i}+\omega_{\mathrm{p}}}-\frac{\exp \left[i\left(\Delta \omega_{j, i}-\omega_{\mathrm{p}}\right) t\right]-1}{\Delta \omega_{j, i}-\omega_{\mathrm{p}}}\right\}
$$

Apparently, the Fourier transform should have three peaks in agreement with those in Fig. 3d. If we substitute equation (5) into equations (1) and (2), besides the oscillations at the frequency $\omega_{\mathrm{p}}$, there are extra oscillating terms at the frequencies $\Delta \omega_{j, i}$. These off-resonant oscillations have different $\Delta \omega_{j, i}$ and thus cannot add up in sync, which results in the irregular oscillations shown in Fig. 3b. To have synchronized plasmon oscillations, the $\omega=0$ peak in the Fourier transform must disappear, which is a hallmark of the plasmon mode. The disappearance can only exist with interactions between different transitions.

In refs 23,42 , when there are no electron-electron interactions ( $\lambda=0$ in ref. 23), the susceptibility $\chi$ equals to that of the non-interacting system $\chi^{0}$. Thus, their model system with no electron-electron interactions is exactly the same as our non-interacting system. The absorption spectrum of the non-interacting system is exactly the DFT result shown in Fig. 1b. (Reference 23) showed that the plasmon frequency significantly increases as $\lambda$ increases. Similarly, we find that the main absorption peak of the real system $(\lambda=1)$ is $\sim 1.9 \mathrm{eV}$ higher than that of the non-interacting system $(\lambda=0)$.

In the discussion above, we have used $i=253$ and 292 with $j=$ LUMO to represent the two types of transitions, respectively. All other transitions can also be classified into the two distinct types. The $\left|C_{j, i}(t)\right|^{2}$ for several other states are shown in the Supplementary Fig. 3 and discussed in Supplementary Note 3.

Here we summarize the analysis above. Both the plasmon and single-particle excitations are described by band to band transitions (equation (1)) in TDDFT. The single-particle excitation is the resonant transition (off-resonant transitions cannot be efficiently excited as single-particle excitations), and equation (1) is rewritten as equation (3); the plasmon is constituted of synchronized off-resonant transitions (the rotating-frame approximation cannot be used), and equation (1) is rewritten as equation (4). The disappearance of the zerofrequency peak in the Fourier transform of $C_{j, i}(t)$ due to the electron-electron interaction is a hallmark of the plasmon. For single-particle excitations, the charge density oscillation comes from the phase factor interference between $\phi_{i}$ and $\phi_{j}\left(\Delta \omega_{j, i} \approx \omega_{\mathrm{p}}\right)$ and $C_{j, i}(t)$ does not oscillate; for plasmons the oscillation comes from the $C_{j, i}(t)$ amplitude oscillations. Each transition $(j, i)$ can either belong to plasmon (off-resonant) or single-particle (nearresonant), but cannot belong to both. This provides an unambiguous way to sort out the plasmon from single-particle excitations.

Energy transfers in $\mathbf{A g}_{\mathbf{5 5}}$. We have calculated the energy spectrum of $\left|C_{j, i}(t)\right|^{2}$, defined as $S_{C}(\omega, t)=\sum_{j, i}\left|C_{j, i}(t)\right|^{2} \delta\left(\omega-\left|\omega_{j}-\omega_{i}\right|\right)$ ( $j$ and $i$ denote unoccupied and occupied states, respectively), which represents the number of transitions at the energy $\omega$ for a given time $t$. For the real system, as shown in Fig. $3 e, S_{C}(\omega, t)$ has peaks around $1.5-2.5 \mathrm{eV}$ at $t=17 \mathrm{fs}$. Those off-resonant transitions constitute the plasmon. At $t=39 \mathrm{fs}$, those off-resonant peaks disappear, and the resonant peak is strengthened, showing that the plasmon has been converted into resonant single-particle excitations (hot carriers). From $t=17$ to $39 \mathrm{fs}$, the laser is off. Thus, the hot carriers are generated completely by the plasmon. For the non-interacting system (Fig. 3f), $S_{\mathrm{C}}(\omega, t)$ shows much smaller peaks around $1.5-2.5 \mathrm{eV}$ (no plasmons), while the main peak always appears at the resonant frequency (single-particle excitations). There is no change in $S_{\mathrm{C}}(\omega, t)$ from 17 to $39 \mathrm{fs}$ because there are no laser nor electron-electron interactions.

We have also calculated $\Delta O_{j}(t)$ defined by equation (8) in Methods section, which is the occupation change on the $j$ th eigen state. The numbers of excited electrons (positive $\Delta O_{j}(t)$ ) on all eigen states are shown in Fig. 4 a. Because the occupation change on an eigen state $j$ can have both resonant and off-resonant 
a

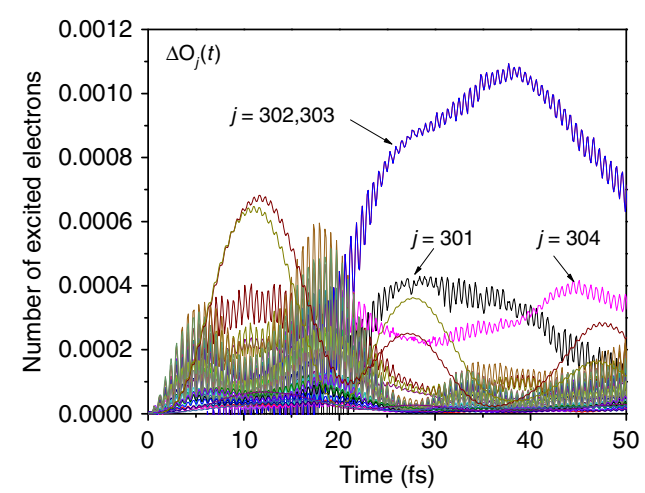

C

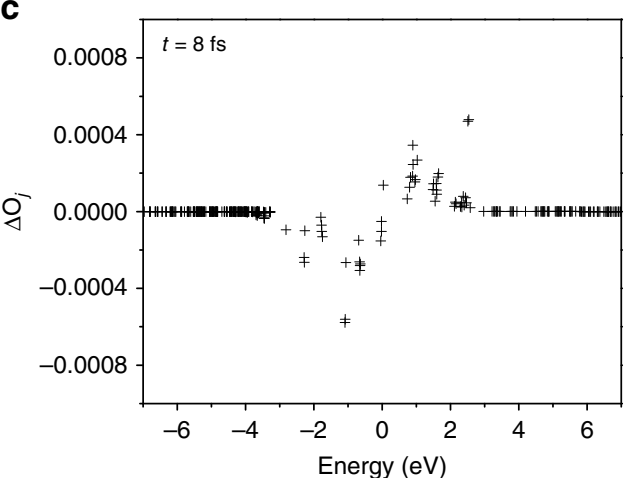

e

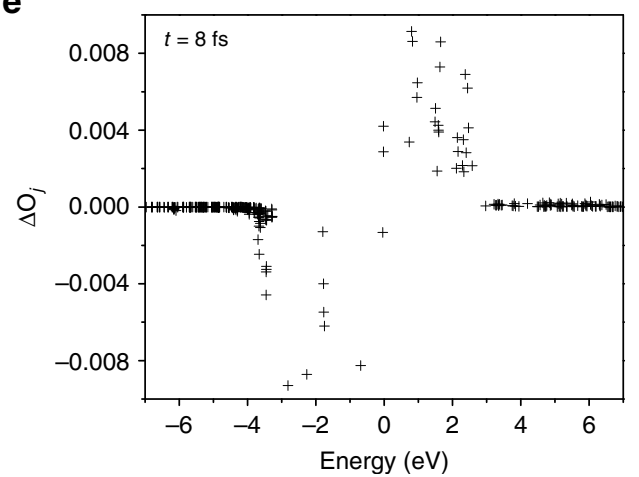

b

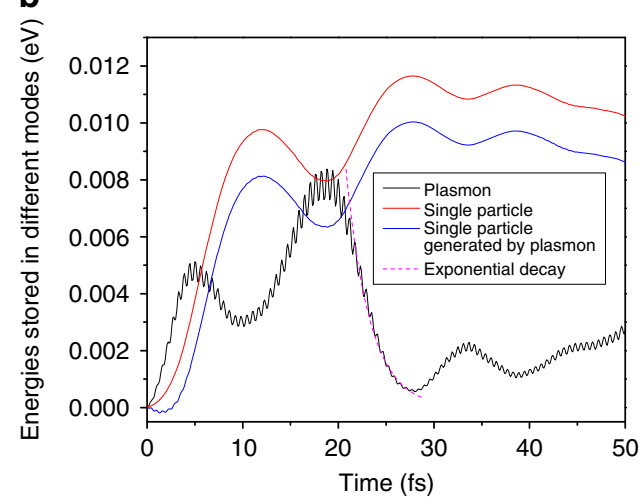

d

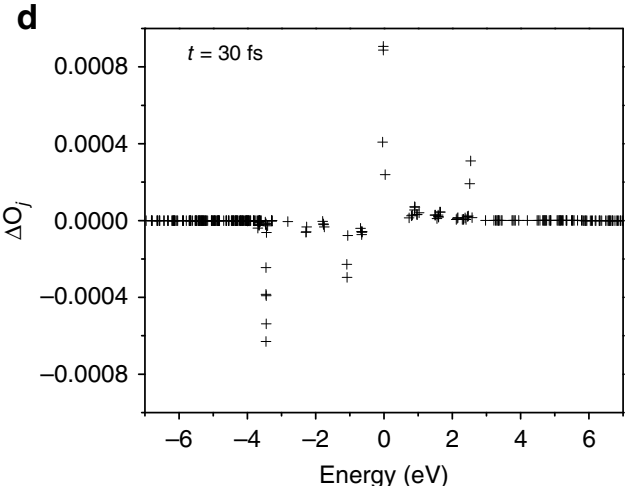

$\mathbf{f}$

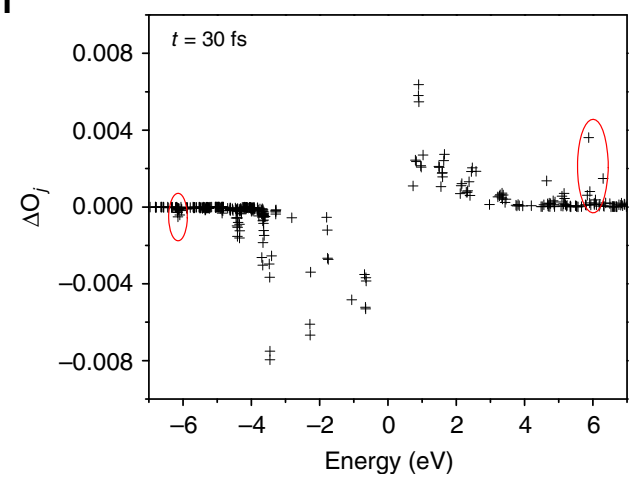

Figure 4 | The time-dependent excitations in $\mathbf{A g}_{\mathbf{5 5}}$. (a) The number of excited electrons on every eigen state as a function of time. Several important eigen states are labelled. The major excitations are onto the 302nd and 303rd eigen states. (b) The energies stored in the plasmon and single-particle excitations. The blue line is the single-particle energy generated by the plasmon, which is defined as the energy difference in the single-particle mode between the real system (rt-TDDFT simulation) and the non-interacting system (in which plasmons cannot exist) under the same laser illumination. The pink-dashed line is an exponential decay of $\exp \left(-\omega_{\mathrm{p}} t / \mathrm{Q}\right)$ with $Q=13.3$. (c,d) The number of excited electrons (positive) and holes (negative) as a function of the corresponding eigen energy with respect to the Fermi energy at $t=8$ and $30 \mathrm{fs}$. At $8 \mathrm{fs}$, most excitations are around the Fermi energy and at $30 \mathrm{fs}$ the excitations are concentrated in a few sharp peaks. (e, f) The number of excited carriers under a strong laser illumination. We observe some high-energy excitations at $30 \mathrm{fs}$ (circled by red), which indicates nonlinear effects.

transitions from different states $i, \Delta O_{j}(t)$ is a combination of both slowly varying and rapidly oscillating components as shown in Fig. $4 \mathrm{a}$, different from $C_{j, i}(t)$ in Fig. 3. We find the major excited electrons are on the eigen states around the Fermi energy (for example, the 302nd and 303rd eigen states) after $20 \mathrm{fs}$. Thus, the single-particle excitations (resonant to the plasmon frequency) from low-energy $d$-states to the eigen states around the Fermi energy are the major plasmon decay (hot-carrier generation) channels.

Our analysis allows us to separate the energies stored in the plasmon and hot carriers (see Methods section), as shown in Fig. 4b. We also plot the single-particle energy generated by the plasmon, which is defined as the energy difference in the single-particle mode between the real system and the non- interacting system (no plasmons) under the same laser. After the laser is turned on at $t=0$, the light energy is absorbed by both the plasmon and resonant single-particle (hot-carrier) excitations, with almost equal rates. Compared with the non-interacting system, the single-particle energy of the real system is lower at first (the blue line is negative), indicating that less hot carriers are generated at first due to the plasmon. After $3 \mathrm{fs}$, the blue line becomes positive, indicating more hot carriers are generated due to the plasmon. After the laser fades off at $t=10 \mathrm{fs}$, the energy in the plasmon mode does not decay immediately, and we even observe a small single-particle to plasmon energy transfer around $t=17 \mathrm{fs}$. From 20 to $30 \mathrm{fs}$, the plasmon energy starts to decay and is almost completely transferred into single-particle excitations. The speed of 
the decay agrees with previous experiments that the plasmon decay timescale is 1-100 fs (refs 11,36). By fitting the decay shoulder with an exponential function $\exp \left(-\omega_{\mathrm{p}} t / Q\right)$, we obtain $Q=13.3$ (the pink-dashed line in Fig. 4b). Without the plasmon (the noninteracting system), the final energy stored in hot carriers is only $\sim 15 \%$ of that with the plasmon, showing that the plasmon can enhance the hot-carrier generations by $\sim 6.6$ times in $\mathrm{Ag}_{55}$. In a recent experiment, Zheng et al. ${ }^{45}$ separated the plasmon-induced and photoexcited hot carriers in $\mathrm{Au}$ nanowires. With the transverse electric polarized laser, the plasmon was excited and the carriers excited by both plasmon and photons were collected through an Ohmic device; with the transverse magnetic polarized laser, the plasmon was not excited and only the carriers excited by photons were collected. In Fig. 3 of ref. 45, the ratio of excited carriers (transverse electric:transverse magnetic) is about 5:1 at the plasmon frequency. This measured plasmon-induced hot-carrier enhancement is in agreement with our result (about 6.6:1). Although the experiment was performed on $\mathrm{Au}$ systems, the agreement provides support for our results. We have performed the rt-TDDFT simulation up to $400 \mathrm{fs}$ (Supplementary Fig. 4. and Note 4), and do not observe strong energy back-flows from hot carriers to plasmon.

The hot-carrier energy distributions at $t=8$ and $30 \mathrm{fs}$ are shown in Fig. $4 \mathrm{c}$,d. At $t=8 \mathrm{fs}$, there are broad excitations around the Fermi energy, indicating the plasmon excitation. At $t=30 \mathrm{fs}$, as the plasmon is decayed to hot carriers, the excited electrons and holes are concentrated in a few sharp peaks. These peaks can be classified into two groups. The major hot holes are around $-3.6 \mathrm{eV}$ with the corresponding electrons around the Fermi energy ( $d$ to $s$ transitions). There are fewer hot holes at $-1.1 \mathrm{eV}$ with the corresponding hot electrons at $2.5 \mathrm{eV}$ ( $s$ to $p$ transitions). In a recent perturbation calculation of plasmon-induced hotcarriers in bulk Ag (ref. 24), the sharp peaks of the hot-carrier energy distribution were also observed. The major surfaceplasmon-induced hot holes are at $-3.6 \mathrm{eV}$ with hot electrons around the Fermi energy ${ }^{24}$, which is in excellent agreement with our results. They also observed other hot holes at -0.1 and hot electrons at $3.6 \mathrm{eV}$, which qualitatively but not quantitatively agrees with our results. This is probably because the nanostructure strongly affects the nonlocalized $s$ and $p$ states but does not strongly affect the localized $d$-states.

Using rt-TDDFT, we can also simulate nonlinear effects, by increasing the laser field by 10 times. The calculated $\Delta O_{j}$ at $t=8$ and $30 \mathrm{fs}$ are shown in Fig. $4 \mathrm{e}, \mathrm{f}$. At $t=8 \mathrm{fs}$, the excitations are similar to those in Fig. 4c, indicating the plasmon excitation. However, at $t=30 \mathrm{fs}$, we observe some high-energy excitations around $\pm 6 \mathrm{eV}$ (circled by red in Fig. 4f), which were not observed in Fig. 4d. Because the high-energy excitations do not exist before the laser fades off ( $t=8 \mathrm{fs}$ in Fig. 4e), they are not due to the nonlinear laser excitation. The energies of those carriers (about $6 \mathrm{eV}$ ) are much greater than the plasmon frequency $(3.6 \mathrm{eV})$, and thus the high-energy carriers are likely due to double excitations by the plasmon. The simulation predicts highenergy hot-carrier generations.

Tuning the energy transfers. As mentioned above, the major hot-carrier generations in $\mathrm{Ag}_{55}$ are from the low-energy $d$-states to the eigen states around the Fermi energy (for example, 302nd and 303rd states). It is interesting to see what would occur if these transitions are blocked. We first consider the negatively charged $\mathrm{Ag}_{55}^{-}$, which is a closed shell system. All the states $j \leq 303$ are fully occupied, and thus cannot accept electrons from $d$-states. The only important channel is the $d$ to 304th state excitation. The amplitude of the dipole oscillation of $\mathrm{Ag}_{55}^{-}$(Fig. 5a) decays much slower than that of the neutral $\mathrm{Ag}_{55}$ (Fig. 2a). The numbers of excited electrons $\Delta O_{j}(t)$ (Fig. 5b) confirm that the dominant hot electrons are on the 304th state. Because most hot-carrier generation channels are closed, the energy stored in the plasmon decreases slowly (Fig. 5c). After $100 \mathrm{fs}$ the energy stored in the plasmon is still decaying and is not totally transferred to hot carriers. We observe small energy transfers and oscillations between the plasmon and single-particle excitations, and the same oscillations of the dipole moment (Fig. 5a). The charge density differences $\Delta \rho$ (Fig. 5d) at $t=19.2$ and 19.8 fs are similar to those of the neutral $\mathrm{Ag}_{55}$ (Fig. 2b). At $t=39.8$ and $40.4 \mathrm{fs}$, there are still strong charge sloshings on the surface, different from those of the neutral $\mathrm{Ag}_{55}$, which also suggests the slow plasmon decay. We would like to stress that the striking change due to one additional electron is related to the electric structure of $\mathrm{Ag}_{55}$. By adding one electron, most hot-carrier generation (plasmon decay) channels are blocked in $\mathrm{Ag}_{55}^{-}$, and thus the plasmon decay becomes much slower. However, for large nanoclusters, there may be many partially occupied states around the Fermi energy and thus adding one electron may not block the major plasmon decay channel or dramatically affect the dynamics.

In $\mathrm{Ag}_{55}^{-}$, the resonant single-particle $d$ to $s$ excitations have not been completely blocked (the $d$ to 304th excitation exists). To change the system more dramatically, one may change the plasmon or single-particle frequencies. Experimentally, it can be realized by alloying with other metals, changing the nanocluster size/shape, or using some dielectric mediums. Theoretically, by further modifying the pseudopotential (see Methods section), we can artificially push down the $d$-state energies, making the $d$ to $s$ transition energies much greater than the plasmon frequency. Thus, all the single-particle $d$ to $s$ excitations become impossible. First, we observe an intriguing oscillation of the dipole amplitude (Fig. 6a). $\Delta O_{j}(t)$ (Fig. 6b) still shows some single-particle excitations, from the 293rd to 332nd and 333rd eigen states ( $s$ to $p$ excitations). However, their transition energies are slightly off-resonant from the plasmon frequency by $\sim 0.2 \mathrm{eV}$, and $\Delta O_{j}$ exhibits Rabi oscillations at the frequency $\Delta \omega_{j, i}-\omega_{\mathrm{p}}$. We also observe a cyclic energy transfer between the plasmon and singleparticle excitations at the Rabi frequency (Fig. 6c). Different from the $\mathrm{Ag}_{55}^{-}$case, the average energies stored in the plasmon and single-particle excitations do not change significantly with time. The charge density differences $\Delta \rho$ (Fig. 6d) at $t=19.2$ and $19.8 \mathrm{fs}$ (more energy is stored in the plasmon) show stronger charge localization on the surface than those at $t=41.4$ and $42.0 \mathrm{fs}$ (more energy is stored in the hot carriers). Around $40 \mathrm{fs}$, there is still charge localized on surface, because the plasmon is not fully decayed. Although Rabi oscillations in two-level systems are well known, it is interesting to observe Rabi oscillations between a collective plasmon mode and individual hot carriers. In the original $\mathrm{Ag}_{55}$ (Fig. $4 \mathrm{~b}$ and Supplementary Fig. 4), we also observe some weak oscillations. However, the regular and complete energy backflow from the single-particle excitations to the plasmon, as described by the resonant Rabi oscillation, is not observed. In plasmonic systems, when the plasmon resonance overlaps with the single-particle interband transitions, the plasmon becomes highly damped and vice versa. This conclusion was achieved by previous model system calculations ${ }^{46}$, and agrees with our results. The predicted Rabi oscillation may be observed experimentally using the real-time optical technique as described in the recent work by Mittal et al. ${ }^{36}$, where long-living (up to $80 \mathrm{fs}$ ) polarization beatings due to two plasmon modes were measured.

Phonon effects. The simulations above have only included plasmon dampings through hot-carrier generations within TDDFT. We have not included the electromagnetic radiation. Reference 47 shows that the radiative effect is very weak, especially for small $\mathrm{Ag}$ 
a
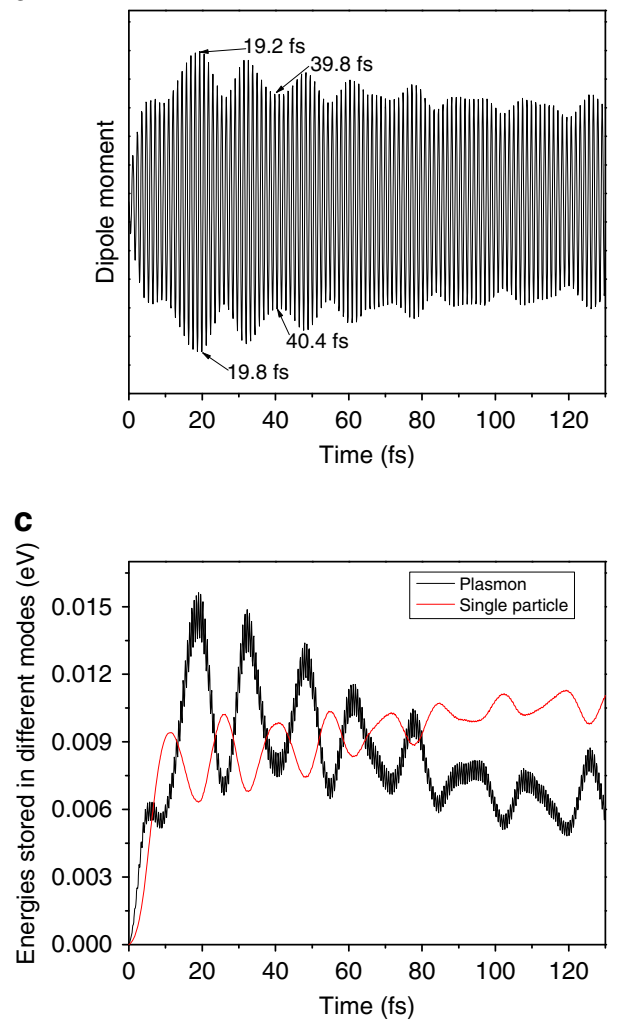

b

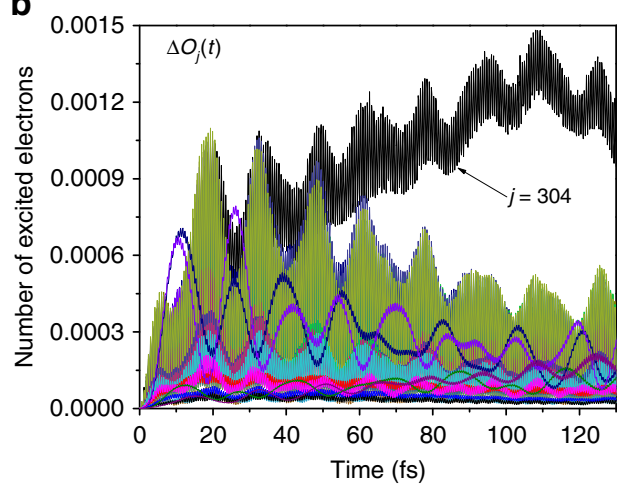

d
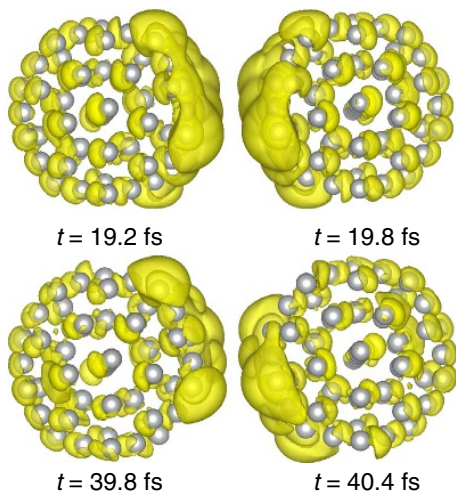

Figure 5 | The time-dependent results for the negatively charged nanocluster. (a) The dipole moment shows a much slower decay compared with the neutral $\mathrm{Ag}_{55}$ (Fig. 2a). (b) The number of excited electrons $\Delta O_{j}(t)$. The major electron excitations are onto the 304th eigen state. The excitations onto the 301st-303rd eigen states shown in Fig. 4 a do not exist. (c) The energies stored in the plasmon mode and single-particle excitations. The energy transfer from the plasmon to single-particle excitations is also much slower compared with Fig. $4 \mathrm{~b}$, which indicates the slow plasmon decay, because the main single-particle $d$ to s excitation channels resonant to the plasmon are blocked in $\mathrm{Ag}_{55}^{-}$. (d) The charge density difference $\Delta \rho$ at time $t$ as labelled in a, which corresponds to the local maximum or local minimum of the dipole moment. Compared with Fig. $2 b$, the significant charge sloshing on the surface still exists around $40 \mathrm{fs}$, which shows the plasmon is not fully decayed.

nanoclusters, and thus can be safely ignored. Another plasmon damping mechanism is the electron-phonon interactions. Previously people believed the electron-phonon interactions can only occur in the timescale of pico-second ${ }^{11}$. However, a recent theoretical work by Bernardi et al. ${ }^{48}$ showed that the phononassisted carrier scattering can occur in tens of femtoseconds for bulk systems. It is thus interesting to investigate the phononassisted damping in nanoclusters. We have performed the rtTDDFT Ehrenfest dynamics simulations. These simulations include the atomic movements following the $a b$ initio forces in the rt-TDDFT, and are able to describe the electron-phonon effects explicitly. The results for neutral $\mathrm{Ag}_{55}$ and $\mathrm{Ag}_{55}$ without resonant hot-carrier generation channels are shown in Fig. 7. Both the dipole moment and the energies stored in both modes are almost the same as those in the simulations without electronphonon interactions, although the simulations with electronphonon interactions indeed show slightly faster decays. Our results show that the electron-phonon interactions only have minor effects in the nanocluster. This is probably because in bulks the energy conservation can always be satisfied and low-energy phonons can induce electron transitions, but it is not the case in nanoclusters with discrete electronic states.

\section{Discussion}

We have performed rt-TDDFT simulations on the $\mathrm{Ag}_{55}$ nanocluster. Our rt-TDDFT simulations include the electronelectron interactions and electron-phonon interactions (using the
Ehrenfest dynamics). The plane-wave basis set allows accurate descriptions of excited states. We do not include the electromagnetic radiations because they are extremely small in Ag nanoclusters. The simulations are under DFT and Kohn-Sham orbital framework, and do not explicitly use many-body wave functions. We propose an approach to distinguish the plasmon from single-particle excitations based on band to band transition coefficients $C_{j, i}(t)$. We find that the collective plasmon consists of off-resonant transitions with $C_{j, i}(t)$ oscillating in their amplitudes at the plasmon frequency, whereas the single-particle excitation is characterized by a slowly varying $C_{j, i}(t)$. The disappearance of the $\omega=0$ peak in the Fourier transform of $C_{j, i}(t)$ makes the plasmonrelated transitions different from the conventional independent off-resonant transitions described by the single-particle timedependent perturbation theory, and is a hallmark of the plasmon. In $\mathrm{Ag}_{55}$, the single-particle $d$ to $s$ excitations resonant to the plasmon frequency exist. The plasmon will decay and generate hot carriers, and significantly enhance the hot-carrier generation rates. The main hot-carrier generations are from the low-energy $d$-states to the $s$-states around the Fermi energy. When applying a high-intensity laser, we also observe some nonlinear high-energy excitations. When most hot-carrier generation channels are blocked in $\mathrm{Ag}_{55}^{-}$, the plasmon decays much slower. We also observe Rabi oscillations between the plasmon and single-particle excitations when the resonant single-particle $d$ to $s$ excitations have been completely blocked. Our rt-TDDFT Ehrenfest dynamic simulations show that the electron-phonon interactions do not play important roles in the plasmon decay in nanoclusters. 
a

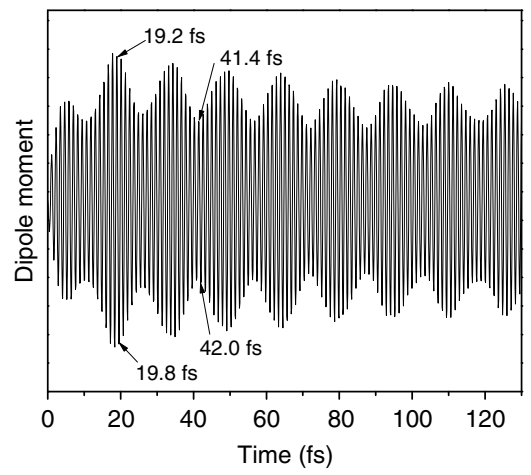

C

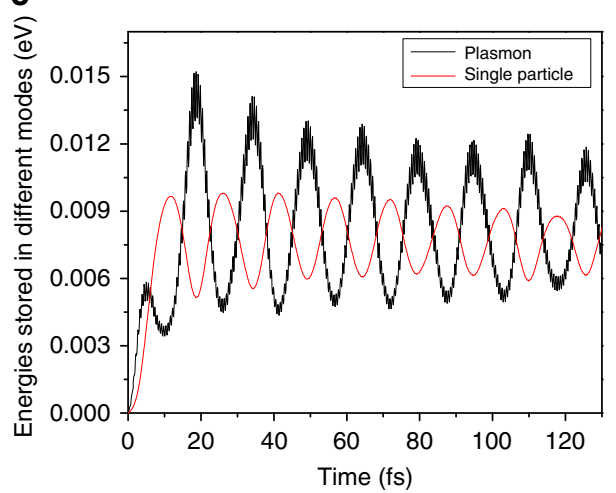

b

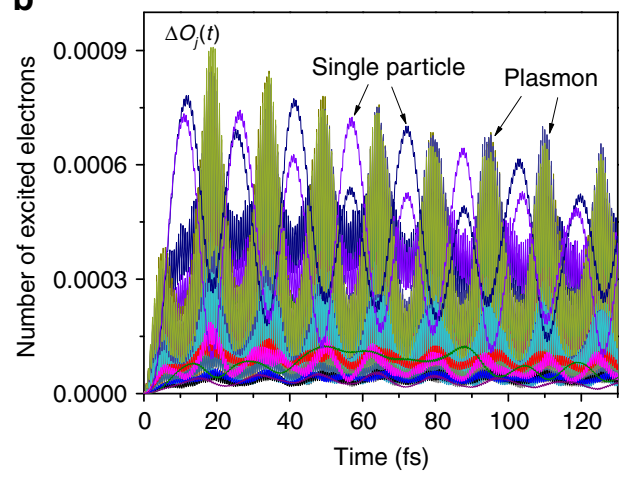

d

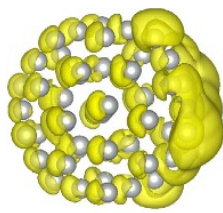

$t=19.2$ fs
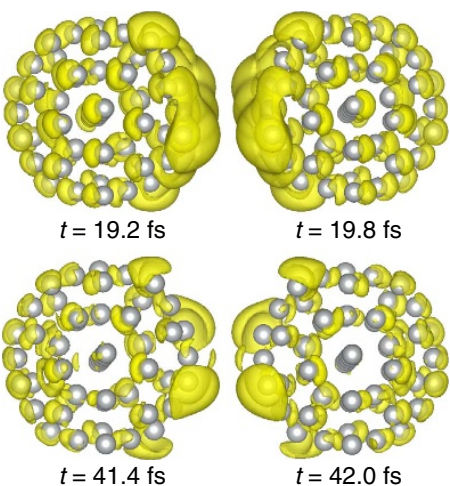

Figure 6 | The time-dependent results for the nanocluster with a modified electronic structure. In this system, the single-particle $d$ to $s$ transitions resonant to the plasmon frequency do not exist. (a) The dipole moment exhibits a Rabi oscillation, and the amplitude of the dipole moment hardly decays. (b) The number of excited electrons $\Delta O_{j}(t)$ also exhibits Rabi oscillations, and the major single-particle excitations are slightly off-resonant. (c) The energies stored in the plasmon mode and single-particle excitations exhibit similar Rabi oscillations. The energy is transferred back and forth between the plasmon and single-particle excitations, and the average energies stored in the plasmon and single-particle excitations do not change significantly with time. (d) The charge density difference $\Delta \rho$ at time $t$ as labelled in $\mathbf{a}$, which corresponds to the local maximum or local minimum of the dipole moment. When more energy is stored in the plasmon mode (19.2 and 19.8 fs), there are significant charge sloshings on the surface; however, when more energy is stored in the single-particle excitations ( 41.4 and $42.0 \mathrm{fs}$ ), there are less charge sloshings on the surface.

\section{Methods}

Computational setups. Our fast rt-TDDFT algorithm ${ }^{31}$ is employed within the local density approximation ${ }^{49}$. The norm-conserving pseudopotential is used and the valence electrons ( $\mathrm{Ag} 4 d^{10}$ and 5s) are expanded using plane-waves with an energy cutoff of $40 \mathrm{Ry}$. Although the plane-wave basis set significantly increases the computational cost compared with the local orbital basis set, it can describe the high-energy excited states and charge responses more accurately, which is critical for the plasmon study. The Brillouin zone integration is sampled with the $\Gamma$ point only for the isolated $\mathrm{Ag}_{55}$ cluster. DFT cannot correctly predict the energies of the $\mathrm{Ag} d$-states, which may affect the accuracy of the plasmon calculations ${ }^{28,50}$. To modify the energy of the $\mathrm{Ag} d$-states, we add a Gaussian term to the $d$-channel of the Ag pseudopotential. We have fitted the pseudopotential so that the calculated density of states of the $\mathrm{Ag} d$-bands agrees well with the experimental X-ray photoemission spectroscopy data ${ }^{35}$, as shown in Supplementary Fig. 1.

In experiments, the $\mathrm{Ag}_{55}$ adopts the icosahedral structure ${ }^{32,33}$. Therefore, we use the icosahedral model, and optimize the atomic coordinates within DFT to get the ground-state structure for the rt-TDDFT simulations. We have also calculated the truncated face-centred cubic structure of $\mathrm{Ag}_{55}$, and the simulated timedependent results have similar features to those of the icosahedral structure, as shown in Supplementary Fig. 5 and Note 5. We have also calculated $A_{55}$, which is shown in Supplementary Fig. 6, but it does not have a clear plasmon peak.

In our simulations, we explicitly consider the atomic structure of the $\mathrm{Ag}_{55}$ nanocluster, and treat the $\mathrm{Ag} 4 d$ electrons as valence electrons. Thus, our simulations give more accurate descriptions of the system than the studies based on jellium models.

When calculating the density of states, the DFT absorption spectrum, and $S_{\mathrm{C}}(\omega$, $t$ ), we broaden the Dirac $\delta$ function using a Gaussian function with a s.d. of $0.1 \mathrm{eV}$.

Plasmon decay channels. The rt-TDDFT simulation explicitly includes the electron-electron interaction, which is critical for the plasmon excitation and plasmon-induced hot-carrier generations. The rt-TDDFT does not include the radiative damping. Previous study shows that the radiative effect is very weak for small Ag nanoclusters, so it is safe to ignore it ${ }^{47}$. The electron-phonon interactions can be explicitly included by using the Ehrenfest dynamics (which is beyond the Born-Oppenheimer approximation). At first, we have fixed the atomic positions in the simulations to focus on the effects of electron-electron interactions. Previous studies show that the electron-phonon interactions are on the timescale of picoseconds ${ }^{11}$, and thus are not important for plasmons. In the last part (Fig. 7), we have included the atomic movements at $50 \mathrm{~K}$ using the Ehrenfest dynamics to show that the electron-phonon interactions only have minor effects. Such a low temperature is typical for $\mathrm{Ag}_{55}$ in experiments due to the high mobility of the nanocluster ${ }^{33}$.

TDDFT algorithm and related formulas. In our rt-TDDFT simulations, the timedependent electronic state $\psi_{i}(t)$ at $t=0$ is occupied according to the ground-state Fermi-Dirac occupation number $O_{i}^{0}$, and the $O_{i}^{0}$ will not be changed with time. The time-dependent wave functions $\psi_{i}(t)$ are always orthogonal to each other. To evolve $\psi_{i}(t)$ with time, we expand it onto the adiabatic eigen states $\phi_{j}(t)$ (which satisfies $H[\rho(t), t] \phi_{j}(t)=\omega_{j}(t) \phi_{j}(t)$ for the time-dependent Hamiltonian $\left.H[\rho(r), t]\right)$ :

$$
\psi_{i}(t)=\sum_{j} e^{-i \int_{0}^{t} \omega_{j}\left(t^{\prime}\right) d t^{\prime}} C_{j, i}(t) \phi_{j}(t)
$$

The $C_{j, i}$ is the expansion coefficient which can be calculated using our rt-TDDFT algorithm ${ }^{31}$. The off-diagonal element $C_{j, i}(i \neq j)$ represents the transition from the $i$ th adiabatic eigen state to the $j$ th adiabatic eigen state. We note that $C_{j, i}$ might depend on an arbitrary phase factor due to the non-unique definition from $\phi_{j}(t)$ to $\phi_{j}(t+d t)$. We have removed this arbitrary phase factor by requiring $\left\langle\phi_{j}(t) \mid \phi_{j}(t+d t)\right\rangle$ to be real. Since $\phi_{j}(t)$ can be taken as a real function (without magnetic fields), there will be no Berry phase. The simulation is under DFT and Kohn-Sham orbital framework, and thus it does not explicitly use many-body wave functions. By using our new algorithm, we can set the simulation time step to $0.1 \mathrm{fs}$ $(\sim 100 \text { times greater than that in conventional rt-TDDFT simulations })^{31}$, which allows us to run long-time simulations using plane-wave basis sets. Note, in the 
a

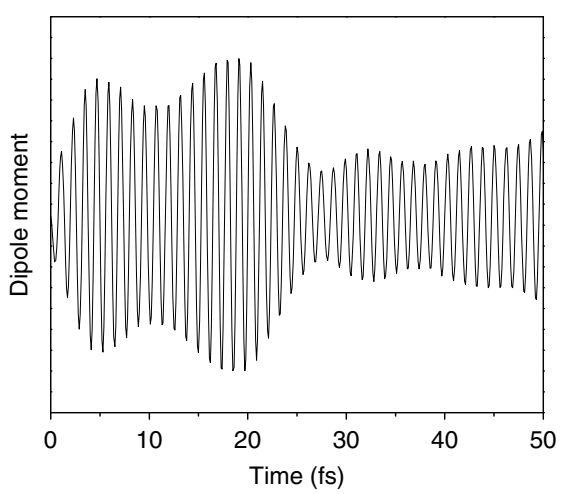

C

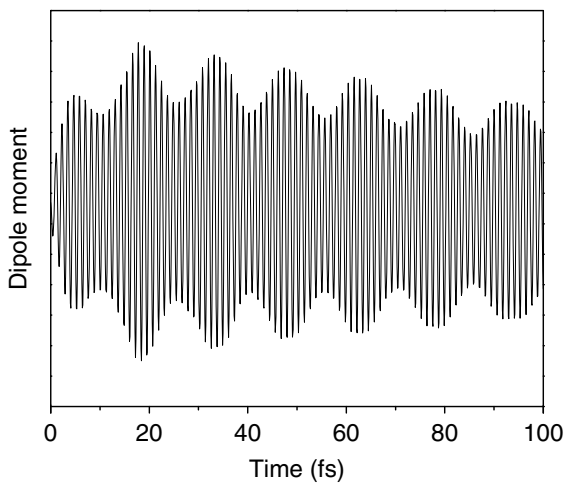

b

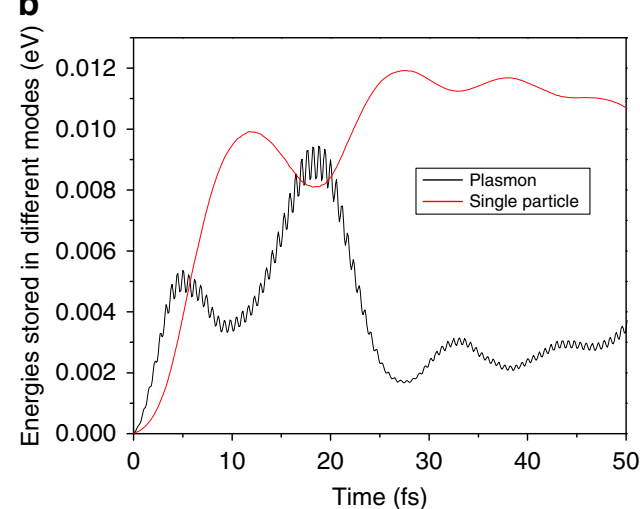

d

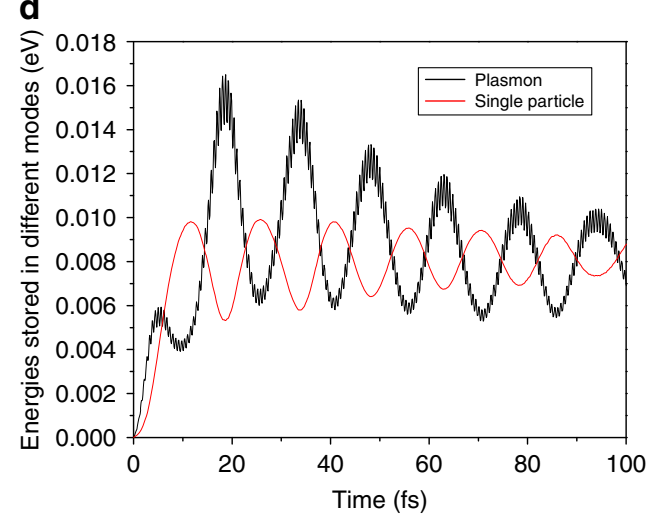

Figure 7 | The rt-TDDFT Ehrenfest dynamics simulations at $\mathbf{5 0} \mathrm{K}$ for neutral $\mathbf{A g}_{55}(\mathrm{a}, \mathrm{b})$ and $\mathbf{A g}_{55}$ with the modified electronic structure $(\mathrm{c}, \mathrm{d})$. In these simulations, the electron-phonon interactions are taken into account explicitly. (a) The dipole moment of the neutral $\mathrm{Ag}_{55}$ is similar to the result without electron-phonon effects shown in Fig. 2a. (b) The energies stored in the plasmon mode and single-particle excitations in the neutral $\mathrm{Ag}_{55}$ are also similar to the results without electron-phonon effects. It should be compared with Fig. $4 \mathrm{~b}$. (c) The dipole moment of the $\mathrm{Ag}_{55}$ with the modified electronic structure (no resonant single-particle $d$ to $s$ excitations). The dipole moment exhibits the Rabi oscillation, similar to the case without electron-phonon effects shown in Fig. 6a. (d) The energies stored in the plasmon mode and single-particle excitations also exhibit the Rabi oscillations when no resonant single-particle excitations exist. It should be compared with Fig. $6 \mathrm{c}$. All these results show that phonons play a minor role in the plasmon decay.

Results part above, we have used constant eigen energies $\omega_{j}$ for the purpose of analysis, as the variations of $\omega_{j}(t)$ are rather small.

The charge density is calculated by

$$
\rho(\mathbf{r}, t)=\sum_{i}\left|\psi_{i}(\mathbf{r}, t)\right|^{2} O_{i}^{0}
$$

Using the coefficients $C_{j, i}$, we define the occupation change $\Delta O_{j}(t)$ on the $j$ th adiabatic eigen state as:

$$
\Delta O_{j}(t)=\left(\sum_{i}\left|C_{j, i}(t)\right|^{2} \times O_{i}^{0}\right)-O_{j}^{0}
$$

The $\Delta O_{j}(t)$ represents the occupation change on the $j$ th adiabatic eigen state at time $t$ with respect to its ground-state occupation $O_{j}^{0}$ due to various electronic excitations. $\Delta O_{j}(t)$ can be either positive or negative. Positive $\Delta O_{j}(t)$ indicates increasing occupations (excited electrons) and negative $\Delta O_{j}(t)$ indicates decreasing occupations (excited holes).

To simulate the laser excitations, we apply an external electric field to the Hamiltonian to mimic the laser pulse

$$
\mathbf{E}(t)=\mathbf{E}_{0} \sin \left(\omega_{1} t\right) \exp \left(-t^{2} / 2 \sigma^{2}\right) / \sqrt{2 \pi} \sigma
$$

The electric field $\mathbf{E}$ is along the $x$ axis. $\sigma, \omega_{1}$ and $\left|\mathbf{E}_{0}\right|$ define the duration, central frequency and amplitude of the electric field, respectively. In this study, we set $\omega_{1}=\omega_{\mathrm{p}}=3.6 \mathrm{eV}, \sigma=6 \mathrm{fs}$ and $\left|\mathbf{E}_{0}\right|=10^{-3}$ Hartree per Bohr.

The polarizability can be obtained from the time-dependent dipole moment of the nanocluster ${ }^{51}$

$$
D(\omega)=\alpha(\omega) E(\omega)
$$

where $D(\omega)$ and $E(\omega)$ are the Fourier transforms of the dipole moment and the electric field, respectively. The absorption spectrum is obtained from the imaginary part of $\alpha(\omega)$.
The energies stored in the plasmon and the single-particle (hot-carrier) modes are estimated as

$$
\begin{aligned}
& E_{\text {plasmon }}(t)=\sum_{(j, i) \in \text { plasmon }}\left|C_{j, i}(t)\right|^{2} \Delta \omega_{j, i} \\
& E_{\text {single }}(t)=\sum_{(j, i) \in \text { single }}\left|C_{j, i}(t)\right|^{2} \Delta \omega_{j, i}
\end{aligned}
$$

where ' $(j, i) \in$ plasmon' and ' $(j, i) \in$ single' indicate the $C_{j, i}(t)$ belongs to the rapidly oscillating transitions and the slowly varying transitions, respectively. For a given $(j, i)$ pair, the $C_{j, i}(t)$ either belongs to the plasmon or single particle, and there is no mixture between the two types. Thus the energies in equation (11) can be calculated unambiguously.

\section{References}

1. Atwater, H. \& Polman, A. Plasmonics for improved photovoltaic devices. Nat. Mater. 9, 205-213 (2010).

2. Novotny, L. \& Hecht, B. Principles of Nano-Optics (Cambridge University Press, 2006).

3. Jensen, L., Aikens, C. M. \& Schatz, G. C. Electronic structure methods for studying surface-enhanced Raman scattering. Chem. Soc. Rev. 37, 1061-1073 (2008).

4. Nie, S. \& Emory, S. Probing single molecules and single nanoparticles by surface-enhanced Raman scattering. Science 275, 1102-1106 (1997).

5. Kuhn, S., Hakanson, U., Rogobete, L. \& Sandoghdar, V. Enhancement of single-molecule fluorescence using a gold nanoparticle as an optical nanoantenna. Phys. Rev. Lett. 97, 017402 (2006).

6. Kreibig, U. \& Vollmer, M. Optical Properties of Metal Clusters (Springer, 1995).

7. Kelly, K., Coronado, E., Zhao, L. \& Schatz, G. The optical properties of metal nanoparticles: the influence of size, shape, and dielectric environment. J. Phys. Chem. B 107, 668-677 (2003). 
8. Linic, S., Christopher, P. \& Ingram, D. B. Plasmonic-metal nanostructures for efficient conversion of solar to chemical energy. Nat. Mater. 10, 911-921 (2011).

9. Knight, M. W., Sobhani, H., Nordlander, P. \& Halas, N. J. Photodetection with active optical antennas. Science 332, 702-704 (2011).

10. Clavero, C. Plasmon-induced hot-electron generation at nanoparticle/metaloxide interfaces for photovoltaic and photocatalytic devices. Nat. Photon. 8, 95-103 (2014).

11. Moskovits, M. The case for plasmon-derived hot carrier devices. Nat. Nanotech. 10, 6-8 (2015).

12. Mukherjee, S. et al. Hot electrons do the impossible: Plasmon-induced dissociation of $\mathrm{H}_{2}$ on Au. Nano Lett. 13, 240-247 (2013).

13. Takahashi, Y. \& Tatsuma, T. Solid state photovoltaic cells based on localized surface plasmon-induced charge separation. Appl. Phys. Lett. 99, 182110 (2011).

14. Wang, F. \& Melosh, N. A. Plasmonic energy collection through hot carrier extraction. Nano Lett. 11, 5426-5430 (2011).

15. Tian, Y. \& Tatsuma, T. Mechanisms and applications of plasmon-induced charge separation at $\mathrm{TiO}_{2}$ films loaded with gold nanoparticles. J. Am. Chem. Soc. 127, 7632-7637 (2005).

16. Brongersma, M. L., Halas, N. J. \& Nordlander, P. Plasmon-induced hot carrier science and technology. Nat. Nanotech. 10, 25-34 (2015).

17. Manjavacas, A. \& Garcia de Abajo, F. Tunable plasmons in atomically thin gold nanodisks. Nat. Commun. 5, 3548 (2014).

18. Scholl, J. A., Koh, A. L. \& Dionne, J. A. Quantum plasmon resonances of individual metallic nanoparticles. Nature 483, 421-427 (2012).

19. Morton, S. M., Silverstein, D. W. \& Jensen, L. Theoretical studies of plasmonics using electronic structure methods. Chem. Rev. 111, 3962-3994 (2011).

20. Aikens, C. M., Li, S. \& Schatz, G. C. From discrete electronic states to plasmons: TDDFT optical absorption properties of $\operatorname{Ag}_{n}(n=10,20,35,56,84,120)$ tetrahedral clusters. J. Phys. Chem. C 112, 11272-11279 (2008).

21. Yan, J., Jacobsen, K. W. \& Thygesen, K. S. Conventional and acoustic surface plasmons on noble metal surfaces: a time-dependent density functional theory study. Phys. Rev. B 86, 241404 (2012).

22. Zuloaga, J., Prodan, E. \& Nordlander, P. Quantum description of the plasmon resonances of a nanoparticle dimer. Nano Lett. 9, 887-891 (2009).

23. Bernadotte, S., Evers, F. \& Jacob, C. R. Plasmons in molecules. J. Phys. Chem. C 117, 1863-1878 (2013).

24. Sundararaman, R., Narang, P., Jermyn, A. S., Goddard, III W. A. \& Atwater, H. A. Theoretical predictions for hot-carrier generation from surface plasmon decay. Nat. Commun. 5, 5788 (2014).

25. Manjavacas, A., Liu, J. G., Kulkarni, V. \& Nordlander, P. Plasmon-induced hot carriers in metallic nanoparticles. ACS Nano 8, 7630-7638 (2014).

26. Townsend, E. \& Bryant, G. W. Plasmonic properties of metallic nanoparticles: The effects of size quantization. Nano Lett. 12, 429-434 (2012).

27. Yan, J., Yuan, Z. \& Gao, S. End and central plasmon resonances in linear atomic chains. Phys. Rev. Lett. 98, 216602 (2007).

28. Kuisma, M. et al. Localized surface plasmon resonance in silver nanoparticles: Atomistic first-principles time-dependent density-functional theory calculations. Phys. Rev. B 91, 115431 (2015).

29. Iida, K., Noda, M., Ishimura, K. \& Nobusada, K. First-principles computational visualization of localized surface plasmon resonance in gold nanoclusters. J. Phys. Chem. A 118, 11317-11322 (2014).

30. Ding, F., Guidez, E. B., Aikens, C. M. \& Li, X. Quantum coherent plasmon in silver nanowires: A real-time TDDFT study. J. Chem. Phys. 140, 244705 (2014).

31. Wang, Z., Li, S.-S. \& Wang, L.-W. Efficient real-time time-dependent density functional theory method and its application to a collision of an ion with a $2 \mathrm{D}$ material. Phys. Rev. Lett. 114, 063004 (2015).

32. Hakkinen, H. et al. Symmetry and electronic structure of noble-metal nanoparticles and the role of relativity. Phys. Rev. Lett. 93, 093401 (2004).

33. Schooss, D. et al. The structures of $\mathrm{Ag}_{55}^{+}$and $\mathrm{Ag}_{55}^{-}$: Trapped ion electron diffraction and density functional theory. Nano Lett. 5, 1972-1977 (2005).

34. Lunskens, T. et al. Plasmons in supported size-selected silver nanoclusters. Phys. Chem. Chem. Phys. 17, 17541-17544 (2015).

35. Barrie, A. \& Christensen, N. E. High-resolution X-ray photoemission spectra of silver. Phys. Rev. B 14, 2442-2447 (1976).

36. Mittal, R., Glenn, R., Saytashev, I., Lozovoy, V. V. \& Dantus, M. Femtosecond nanoplasmonic dephasing of individual silver nanoparticles and small clusters. J. Phys. Chem. Lett. 6, 1638-1644 (2015).
37. Guidez, E. B. \& Aikens, C. M. Origin and TDDFT benchmarking of the plasmon resonance in acenes. J. Phys. Chem. C 117, 21466-21475 (2013).

38. Guidez, E. B. \& Aikens, C. M. Plasmon resonance analysis with configuration interaction. Phys. Chem. Chem. Phys. 16, 15501-15509 (2014).

39. Piccini, G., Havenith, R. W. A., Broer, R. \& Stener, M. Gold nanowires: a time-dependent density functional assessment of plasmonic behavior. J. Phys. Chem. C 117, 17196-17204 (2013).

40. Townsend, E. \& Bryant, G. W. Which resonances in small metallic nanoparticles are plasmonic? J. Opt. 16, 114022 (2014).

41. Krauter, C. M., Schirmer, J., Jacob, C. R., Pernpointner, M. \& Dreuw, A. Plasmons in molecules: Microscopic characterization based on orbital transitions and momentum conservation. J. Chem. Phys. 141, 104101 (2014).

42. Manjavacas, A., Thongrattanasiri, S. \& Garcia de Abajo, F. J. Plasmons driven by single electrons in graphene nanoislands. Nanophotonics 2, 139-151 (2013).

43. Beck, D. E. Self-consistent calculation of the eigenfrequencies for the electronic excitations in small jellium spheres. Phys. Rev. B 35, 7325-7333 (1986).

44. Zeng, J. Quantum Mechanics (Science Press, 2006).

45. Zheng, B. Y. et al. Distinguishing between plasmon-induced and photoexcited carriers in a device geometry. Nat. Commun. 6, 7797 (2015).

46. Marton, J. P. \& Jordan, B. D. Optical properties of aggregated metal systems: Interband transtions. Phys. Rev. B 15, 1719-1727 (1977).

47. Ross, M. B. \& Schatz, G. C. Radiative effects in plasmonic aluminum and silver nanospheres and nanorods. J. Phys. D Appl. Phys 48, 184004 (2015).

48. Bernardi, M., Mustafa, J., Neaton, J. B. \& Louie, S. G. Theory and computation of hot carriers generated by surface plasmon polaritons in noble metals. Nat. Commun. 6, 7044 (2015).

49. Kohn, W. \& Sham, L. J. Self-consistent equations including exchange and correlation effects. Phys. Rev. 140, A1133 (1965).

50. Bae, G.-T. \& Aikens, C. M. Time-dependent density functional theory studies of optical properties of Ag nanoparticles: Octahedra, truncated octahedra, and icosahedra. J. Phys. Chem. C 116, 10356-10367 (2012).

51. Lopata, K. \& Govind, N. Modeling fast electron dynamics with real-time time-dependent density functional theory: application to small molecules and chromophores. J. Chem. Theory Comput. 7, 1344-1355 (2011).

\section{Acknowledgements}

This material is based on the work performed by the Joint Center for Artificial Photosynthesis, a DOE Energy Innovation Hub, supported through the Office of Science of the US Department of Energy under Award number DE-SC0004993. We used the resource of National Energy Research Scientific Computing center (NERSC) located in Lawrence Berkeley National Laboratory. We also used computational resources of the Oak Ridge Leadership Computing Facility at the Oak Ridge National Laboratory, which is supported by the Office of Science of the DOE under contract No. DE-AC05-00OR22725, with computational time allocated by the Innovative and Novel Computational Impact on Theory and Experiment project.

\section{Author contributions}

J.M. and L.-W.W. contributed to all aspects of this work, and Z.W. contributed to modifying the TDDFT code.

\section{Additional information}

Supplementary Information accompanies this paper at http://www.nature.com/ naturecommunications

Competing financial interests: The authors declare no competing financial interests.

Reprints and permission information is available online at http://npg.nature.com/ reprintsandpermissions/

How to cite this article: Ma, J. et al. Interplay between plasmon and single-particle excitations in a metal nanocluster. Nat. Commun. 6:10107 doi: 10.1038/ncomms10107 (2015).

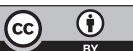

This work is licensed under a Creative Commons Attribution 4.0 International License. The images or other third party material in this article are included in the article's Creative Commons license, unless indicated otherwise in the credit line; if the material is not included under the Creative Commons license, users will need to obtain permission from the license holder to reproduce the material. To view a copy of this license, visit http://creativecommons.org/licenses/by/4.0/ 